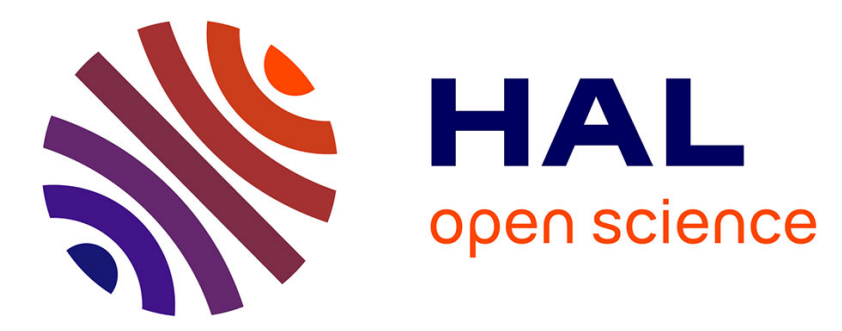

\title{
Decoherence and protection of entanglement of a system of three qubits driven by a classical Gaussian distributed fluctuating field
}

Lionel Tenemeza Kenfack, Martin Tchoffo, Lukong Cornelius Fai, Lionel

Tenemeza Kenfack

\section{To cite this version:}

Lionel Tenemeza Kenfack, Martin Tchoffo, Lukong Cornelius Fai, Lionel Tenemeza Kenfack. Decoherence and protection of entanglement of a system of three qubits driven by a classical Gaussian distributed fluctuating field. 2018. hal-01715803

\section{HAL Id: hal-01715803 \\ https://hal.science/hal-01715803}

Preprint submitted on 23 Feb 2018

HAL is a multi-disciplinary open access archive for the deposit and dissemination of scientific research documents, whether they are published or not. The documents may come from teaching and research institutions in France or abroad, or from public or private research centers.
L'archive ouverte pluridisciplinaire HAL, est destinée au dépôt et à la diffusion de documents scientifiques de niveau recherche, publiés ou non, émanant des établissements d'enseignement et de recherche français ou étrangers, des laboratoires publics ou privés. 


\title{
Decoherence and protection of entanglement of a system of three qubits driven by a classical Gaussian distributed fluctuating field
}

\author{
Lionel Tenemeza Kenfack * Martin Tchoffo \\ Laboratoire de Matière Condensée, D'électronique et de Traitement du Signal (LAMACETS), \\ Departement de Physique, Faculté des Sciences, Université de Dschang, PO Box: 67 Dschang, Cameroun.
}

(Dated: February 23, 2018)

\begin{abstract}
We consider a system of three uncoupled entangled qubits undergoing a decoherence process (DP) induces by a classical environmental noise portrayed by a Gaussian distributed fluctuating field with either Ornstein-Uhlenbeck (OU) or Gaussian (G) autocorrelation function. The impacts of such a DP on the entanglement of the qubits are analyzed in detail when they are initially prepared in the GHZ- and W-type states and coupled to the fluctuating field in three different scenarios namely, common, independent and mixed environment(s). We found that: (i) the way the qubits are coupled to the noise as well as their initial state play an important role towards the protection of entanglement; (ii) there are optimal parameters which permit to delay or totally avoid the disentanglement of the qubits; (iii) regardless of the qubit-noise coupling (QNC) scenario and initial state of the qubits considered, the OU noise is more fatal to the survival of entanglement than the G one. Concretely speaking, we show that, regardless of the QNC scenario and the nature of the noise considered, the DP disentangles the qubits more quickly when they are initially prepare in the W-type states than in the GHZ-type ones. On the other hand, we show that when the qubits are set in the $\mathrm{W}$-type states, the disentanglement occurs more rapidly in the common environment scenario followed by the mixed environments one than in the independent environments one. However, the situation is completely reversed when the qubits are set in the GHZ-type states.
\end{abstract}

PACS numbers: 03.67. a, 03.65.Yz, 05.40. a

Keywords: Qubits; Classical noise; Entanglement; Fluctuating field

\section{INTRODUCTION}

The development of quantum technologies is based on the creation and manipulation of the quantum properties of quantum systems such as quantum correlations (entanglement or superposition of quantum states and discord). Consequently, entanglement is nowadays recognizes as the most important and indispensable form of quantum correlations for performing quantum computations 2] or other task such as, quantum teleportation [3], quantum dense coding [4] and quantum cryptography [5]. In quantum mechanics, every system always interacts with its surrounding environment and this is known as decoherence. Unfortunately, decoherence is the most obstacle to the reliable exploitation of the quantum properties of a quantum system. In point of, quantum entanglement and other correlations are very brittle and might be pulled down by the action of decoherence. Hence, exploring strategies to overcome the detrimental effects of decoherence is very important for the development of quantum technologies and this may be possible by a deep understanding of the decoherence mechanisms in quantum systems together with suitable environment engineering.

Decoherence may be simulated classically or quantum mechanically. The classical simulation, also known as stochastic modeling is often used to describes environments with many degrees of freedom and/or structured noise spectrum [6, 8], or to describes quantum systems under the action of a classical random field [7, 8]. In particular, the stochastic modeling of the environment is often the main effective tool available in physical, biological and social networks [9-12] to describe complex systems. On the other hand, in a more realistic situation, environment surrounding a quantum system is constituted by a collection of many random fluctuators [6, 7]. Thus, stationary and non-stationary Gaussian or non-Gaussian processes are often used to characterize the response of a quantum system under the action of an external environment. On the other hand, many experimental investigations showed that quantum systems often interact with classical forms of noise, especially Gaussian noise 13 -15. Although the decoherence is often described quantum mechanically, it has been shown that even certain quantum decoherence models may be in an efficient way described by their equivalent classical models [34 39]. Besides, it has been demonstrated that modeling the environment classically allows a proper description of the dynamics in presence of memory effects, without resorting to approximated quantum master equations and that the use of classical stochastic elds enables the study of a larger class of processes standard quantum equations are not able to represent [1].

In recent year, the dynamics of quantum correlations in the situation where quantum systems are affected by environments describes classically (i.e., model by means of a classical Gaussian or non-Gaussian distributed random 
field) has been an active area of research [6, 7, 16 33] and many interesting results have been obtained. In particular, it has been shown that when three qubits are coupled to an external classical noise such as random telegraph noise [16 18], static noise [20] and colored noise [19, 21] in a common environment, quantum correlations among them survive indefinitely. However, it is worth noting that the majority of these works deals with bipartite systems. This is mainly because of lack of computable quantum correlations measure for multipartite systems and also because the calculation difficulties grow up when the number of subsystem of a quantum system increases. Although the effects of Gaussian distributed classical noises on the dynamics of quantum correlations have already been investigated for bipartite systems [6], it extension to multipartite systems appears quite promising since here, quantum correlations derive from a more complex scenario, and, as a consequence, more strange phenomena are expected.

In this paper, we consider a system of three non-interacting qubits coupled with an external classical environment in three different scenarios namely: common, independent and mixed environment(s) (see figure 1 of Refs. [19, 20]). In the first scenario, we assume that the three qubits are coupled to the same environment; in the second scenario, we assume that each qubit is coupled to its own environment while in the third scenario two of them are coupled to the same environment and the remaining one to its own environment. The classical external environment is models as a Gaussian distributed stochastic fields with Ornstein-Uhlenbeck (OU) and Gaussian (G) autocorrelation function. We consider the situation where the noise spectrum of the aforementioned stochastic fields contains frequencies that are larger than the typical frequencies of the qubits so that the system cannot be described as a pure dephasing. Numerical and analytic results for entanglement are presented for GHZ-type and W-type initial states by using the tripartite negativity as entanglement measure. The influences of the qubit-environment configuration as well as some parameters of the stochastic field that models the environment such as its central frequency and its correlation time on the evolution of entanglement are investigated.

The purpose of this paper is to illustrate the impacts of the decoherence process induces by a classical environment portrayed by a Gaussian distributed stochastic field with either Ornstein-Uhlenbeck or Gaussian autocorrelation function on the evolution of entanglement of a system of three noninteracting qubit and find strategies to delay or completely suppress such a decoherence process.

The paper is organized as follows: in section. [I] we present the physical model, introduce the tripartite negativity and describe the Gaussian distributed fluctuating fields that portrayed the external environment. In section. III. we investigate the decoherence process of the external environment. In section. IV] we present the evolution of entanglement and analyze in some detail the impacts of the initial prepared state of the system and the nature of the fluctuating field on the evolution of entanglement. In section. V] we close the paper with some concluding remarks.

\section{PHYSICAL MODEL}

We consider a system of three noninteracting qubit interacting with a classical stochastic field (CSF) either in a commont environment (CE), independent environments (IEs) or mixed environments (MEs). We assume that the noise spectrum of the CSF contains frequencies that are larger than the natural frequency $\epsilon_{0}$ of the qubit. In such a situation, the dominant process induced by the CSF is damping [8] and the single-qubit Hamiltonian may be written as:

$$
\mathcal{H}_{k}(t)=\epsilon_{k, 0} \sigma_{k}^{z}+g_{k}\left[\vartheta_{k}(t) e^{-\imath \omega_{k} t}+\bar{\vartheta}_{k}(t) e^{\imath \omega_{k} t}\right] \sigma_{k}^{x}
$$

where $\epsilon_{k, 0}$ is the natural frequency of the qubit $k, \sigma_{k}^{i},(i=x, z)$ are the Pauli matrices acting on the subspace of qubit $k, g_{k}$ stands for the qubit-environment coupling constant, $\vartheta_{k}(t)$ is a Gaussian distributed CSF with central frequency $\omega_{k}$, with a noise spectrum containing frequencies that are larger than $\epsilon_{k, 0}$ and whose complex conjugate is $\bar{\vartheta}_{k}(t)$. It is worth nothing that $\vartheta_{1}(t)=\vartheta_{2}(t)=\vartheta_{3}(t)$ when the qubits are coupled in a CE, meanwhile $\vartheta_{1}(t) \neq \vartheta_{2}(t) \neq \vartheta_{3}(t)$ when they are coupled in IEs. However, in the case of MEs, we assume that $\vartheta_{1}(t)=\vartheta_{2}(t) \neq \vartheta_{3}(t)$. The full Hamiltonian of the three-qubit system is given by:

$$
\mathcal{H}(t)=\mathcal{H}_{1}(t) \otimes \mathbb{I}_{2} \otimes \mathbb{I}_{3}+\mathbb{I}_{1} \otimes \mathcal{H}_{2}(t) \otimes \mathbb{I}_{3}+\mathbb{I}_{1} \otimes \mathbb{I}_{2} \otimes \mathcal{H}_{3}(t)
$$

where, $\mathbb{I}_{k}$ denotes the identity operator in the subspace of the k's qubit, $\mathcal{H}_{k}(t),(k=1,2,3)$ stands for the single-qubit Hamiltonian whose explicit expression is given in Eq. (1). The model Hamiltonian in Eq. (1) represents the stochastic modeling of one of the most form of noise (phase diffusion) a quantum systems are often suffer. Concretely speaking, the model under consideration may characterizes a spin- $\frac{1}{2}$ particle (whose motional degree of freedom is coupled to its quantum degree of freedom e.g., its spin [8]) suffering a diffusion process in an external field having frequencies that are larger than the natural frequency of the particle.

In this model, we assume that $\vartheta_{k}(t)=\vartheta_{k}^{x}(t)+\imath \vartheta_{k}^{y}(t)$ is described by a Gaussian stochastic process with zero mean 
and diagonal structure of the autocorrelation function. More specifically, we have

$$
\left\{\begin{array}{l}
\mathbb{E}\left[\vartheta_{k}^{x}(t)\right]=\mathbb{E}\left[\vartheta_{k}^{y}(t)\right]=0 \\
\mathbb{E}\left[\vartheta_{k}^{x}\left(t_{1}\right) \vartheta_{k}^{x}\left(t_{2}\right)\right]=\mathbb{E}\left[\vartheta_{k}^{y}\left(t_{1}\right) \vartheta_{k}^{y}\left(t_{2}\right)\right]=\mathrm{K}\left(t_{1}, t_{2}\right), \\
\mathbb{E}\left[\vartheta_{k}^{x}\left(t_{1}\right) \vartheta_{k}^{y}\left(t_{2}\right)\right]=\mathbb{E}\left[\vartheta_{k}^{y}\left(t_{1}\right) \vartheta_{k}^{x}\left(t_{2}\right)\right]=0
\end{array}\right.
$$

Upon assuming $t_{0}=0$, the single qubit evolution operator for a given realization of the CSF $\vartheta_{k}(t)$ may be expressed as:

$$
\mathcal{U}_{k}\left(t, \in_{k, 0}\right)=T \exp \left\{-\imath \int_{0}^{t} \mathcal{H}_{k}(\tau) d \tau\right\}
$$

where $T$ stands for the time ordering operator. In the limit of slowly varying fluctuating field (quasi static regime), the two-time commutator $\left[\mathcal{H}_{k}\left(t_{1}\right), \mathcal{H}_{k}\left(t_{2}\right)\right]$ may be neglected. That is, the single qubit Hamiltonian $\mathcal{H}_{k}(t)$ commutes with itself for different times. In such a situation, the quasi static evolution operator is obtained and its reads:

$$
\mathcal{U}_{k}\left(t, \in_{k, 0}\right)=\exp \left\{-\imath \int_{0}^{t} \mathcal{H}_{k}(\tau) d \tau\right\}=\exp \left\{-\imath\left[\epsilon_{k, 0} t \sigma_{k}^{z}+g_{k}\left(\int_{0}^{t} \eta_{k}(\tau) d \tau\right) \sigma_{k}^{x}\right]\right\}
$$

where we have set $\eta_{k}(\tau)=\vartheta_{k}(\tau) e^{-\imath \omega_{k} \tau}+\bar{\vartheta}_{k}(\tau) e^{\imath \omega_{k} \tau}$. Knowing that

$$
\exp \left[-\imath\left(\alpha \sigma_{x}+\gamma \sigma_{z}\right)\right]=\cos \left(\sqrt{\alpha^{2}+\gamma^{2}}\right) \mathbb{I}-\imath \frac{\sin \left(\sqrt{\alpha^{2}+\gamma^{2}}\right)}{\sqrt{\alpha^{2}+\gamma^{2}}}\left(\alpha \sigma_{x}+\gamma \sigma_{z}\right)
$$

the quasi static evolution operator in Eq. (5) can be written as:

$$
\mathcal{U}_{k}\left(t, \epsilon_{k, 0}\right)=\cos \left(\sqrt{\left(\epsilon_{k, 0} t\right)^{2}+\varphi_{k}^{2}(t)}\right) \mathbb{I}-\imath \frac{\sin \left(\sqrt{\left(\epsilon_{k, 0} t\right)^{2}+\varphi_{k}^{2}(t)}\right)}{\sqrt{\left(\epsilon_{k, 0} t\right)^{2}+\varphi_{k}^{2}(t)}}\left(\epsilon_{k, 0} t \sigma_{x}+\varphi_{k}^{2}(t) \sigma_{z}\right)
$$

where we have introduce the noise phase

$$
\varphi_{k}(t)=g_{k} \int_{0}^{t} \eta_{k}(\tau) d \tau
$$

Since the noise spectrum of the CSF contains frequencies that are larger than $\in_{k, 0}$, we may assume that $\in_{k, 0} \ll$ $g_{k}\left|\eta_{k}(t)\right|, \forall t$, that is, $\epsilon_{k, 0} t \ll \varphi_{k}(t)$. Therefore, from this assumption we can rewrite the single qubit evolution operator in Eq. (7) as:

$$
\mathcal{U}_{k}(t) \approx \cos \varphi_{k}(t) \mathbb{I}-\imath \sin \varphi_{k}(t) \sigma_{k}^{x} \approx\left[\begin{array}{cc}
\cos \varphi_{k}(t) & -\imath \sin \varphi_{k}(t) \\
-\imath \sin \varphi_{k}(t) & \cos \varphi_{k}(t)
\end{array}\right]
$$

It is important to note that the same evolution operator can be obtained without any approximation but rather in the situation were the energy levels of the qubit are degenerated as in Refs. [17, 19, 22]. In other words, we show that when a qubit is couple to a CSF having a spectrum with frequencies that are larger than its natural frequency, its dynamics is approximately the same as the one of a qubit with degenerated energy levels coupled to the same CSF. Hence, the question arising is: is the transition between the two states of the qubit still possible? If yes, how can we control these transitions or the states occupation in such a system? In order to bring an answer to these questions, we compute the survival and transition probability of the qubit and their read:

$$
\mathrm{P}_{s}(t)=\left|\left\langle\uparrow\left|\mathcal{U}_{k}(t)\right| \uparrow\right\rangle\right|^{2}=\frac{1}{2}\left(1+\mathbb{E}[\cos 2 \varphi(t)]_{\varphi}\right)
$$

and

$$
\mathrm{P}_{t r}(t)=\left|\left\langle\uparrow\left|\mathcal{U}_{k}(t)\right| \downarrow\right\rangle\right|^{2}=\frac{1}{2}\left(1-\mathbb{E}[\cos 2 \varphi(t)]_{\varphi}\right)
$$


where the symbol $\mathbb{E}[\ldots]_{\varphi}$ stands for the average over all the possible realizations of the noise phase $\varphi(t)$. Note that the subscript $k$ has been dropped because the qubits are identical. It can easily be seen from Eqs. (10) and (11) that in the absence of noise the survival probability is equal to unit (i.e., the qubit will remains in its initial state) while the transition probability is equal to zero that is the transition between the two degenerated state of the qubit is not possible.

Since the qubits are non-interacting, they evolved independently to each others and the total evolution operator of the system can be written as:

$$
\begin{aligned}
\mathcal{U}(t) & =\mathcal{U}_{1}(t) \otimes \mathcal{U}_{2}(t) \otimes \mathcal{U}_{3}(t)=\cos \varphi_{1}(t) \cos \varphi_{2}(t) \cos \varphi_{3}(t) \mathbb{I}_{8}-\imath \cos \varphi_{1}(t) \cos \varphi_{2}(t) \sin \varphi_{3}(t) \mathbb{I}_{4} \otimes \sigma_{x} \\
& -\imath \sin \varphi_{1}(t) \cos \varphi_{2}(t) \cos \varphi_{3}(t) \sigma_{x} \otimes \mathbb{I}_{4}-\cos \varphi_{1}(t) \sin \varphi_{2}(t) \sin \varphi_{3}(t) \mathbb{I}_{2} \otimes \sigma_{x} \otimes \sigma_{x}- \\
& -\sin \varphi_{1}(t) \cos \varphi_{2}(t) \sin \varphi_{3}(t) \sigma_{x} \otimes \mathbb{I}_{2} \otimes \sigma_{x}-\imath \cos \varphi_{1}(t) \sin \varphi_{2}(t) \cos \varphi_{3}(t) \mathbb{I}_{2} \otimes \sigma_{x} \otimes \mathbb{I}_{2}- \\
& -\sin \varphi_{1}(t) \sin \varphi_{2}(t) \cos \varphi_{3}(t) \sigma_{x} \otimes \sigma_{x} \otimes \mathbb{I}_{2}+\imath \sin \varphi_{1}(t) \sin \varphi_{2}(t) \sin \varphi_{3}(t) \sigma_{x} \otimes \sigma_{x} \otimes \sigma_{x}
\end{aligned}
$$

where we have used the fact that the three qubits are identical to simplify the expressions. Note that $\mathbb{I}_{\ell},(\ell=2,4,8)$ stands for a $\ell \times \ell$ identity matrix. It is worth nothing that the evolution operator in Eq. (12) is fully for the situation where the qubit are coupled to the CSF in IEs. However, the evolution operator for the case of MEs and CE can be easily derived from the IEs one by taking $\varphi_{1}(t)=\varphi_{2}(t)$ and $\varphi_{1}(t)=\varphi_{2}(t)=\varphi_{3}(t)$ respectively.

Upon denoting by $\rho(0)$ the initial state of the three qubits, the time evolved density matrix of the system for a given realization of the noise phase $\varphi_{k}(t)$ reads:

$$
\rho(t)=\mathbb{E}\left[\mathcal{U}(t) \rho(0) \mathcal{U}(t)^{\dagger}\right]_{\left\{\varphi_{k}(t)\right\}}
$$

were $\mathbb{E}[\cdots]_{\left\{\varphi_{k}(t)\right\}}$ stands for the average over all the possible realizations and configurations of the noise phase $\varphi_{k}(t)$. As previously mentioned, we assume that the three qubits are initially prepared in two different well know entangled states namely the GHZ- and W-type states which cannot be transform into each other by any stochastic local operations assisted by classical communication (SLOCC) [40]. They are defined as follows:

$$
\rho_{G H Z}(0)=\frac{q}{2}(|000\rangle+|111\rangle)(\langle 000|+\langle 111|)+\frac{1-q}{8} \mathbb{I}_{8}
$$

and

$$
\rho_{W}(0)=\frac{q}{3}(|001\rangle+|010\rangle+|100\rangle)(\langle 001|+\langle 010|+| 100\rangle)+\frac{1-q}{8} \mathbb{I}_{8}
$$

where the parameter $q \in[0,1]$ stands for the purity of the initial state and $\mathbb{I}_{8}$ is $8 \times 8$ identity matrix.

Upon substituting the expression of $\mathcal{U}(t)$ given in Eq. (12), we find that the evaluation of the density matrix $\rho(t)$ required the evaluation of the averaged terms of the form $\mathbb{E}\left[\cos \left(m \varphi_{k}(t)\right)\right]_{\varphi_{k}}$ and $\mathbb{E}\left[\sin \left(m \varphi_{k}(t)\right)\right]_{\varphi_{k}}$. These terms can be easily computed if we know the characteristics function of the random noise phase $\varphi_{k}(t)$. By definition, the characteristic function of $\varphi_{k}(t)$ is given by:

$$
\mathbb{E}\left[\exp \left(\imath \varphi_{k}(t)\right)\right]_{\varphi_{k}}=\exp \left(\imath \mathbb{E}\left[\varphi_{k}(t)\right]+\frac{1}{2} \mathbb{E}\left[\left(\delta \varphi_{k}(t)\right)^{2}\right]\right)
$$

where $\delta \varphi_{k}(t)=\varphi_{k}(t)-\mathbb{E}\left[\varphi_{k}(t)\right]$.

After straightforward calculation, it is easy to see that

$$
\varphi_{k}(t)=2 g_{k} \int_{0}^{t}\left(\vartheta_{k}^{x}(t) \cos \left(\omega_{k} \tau\right)+\vartheta_{k}^{y}(t) \sin \left(\omega_{k} \tau\right)\right) d \tau=2 g_{k} \int_{0}^{t}\left(\xi_{k}^{x}(\tau)+\xi_{k}^{y}(\tau)\right) d \tau
$$

with $\xi_{k}^{x}(\tau)=\vartheta_{k}^{x}(t) \cos \left(\omega_{k} \tau\right)$ and $\xi_{k}^{y}(\tau)=\vartheta_{k}^{y}(t) \sin \left(\omega_{k} \tau\right)$. Upon substituting the above equation in Eq. (16) we obtain the characteristic function of the noise phase $\varphi_{k}(t)$ as:

$$
\begin{aligned}
\mathbb{E}\left[\exp \left(\imath \varphi_{k}(t)\right)\right]_{\varphi_{k}} & =\exp \left(-\frac{1}{2} \mathbb{E}\left[\left(2 g_{k} \int_{0}^{t}\left(\xi_{k}^{x}(\tau)+\xi_{k}^{y}(\tau)\right) d \tau\right)^{2}\right]\right) \\
& =\exp \left(-\frac{1}{2} \mathbb{E}\left[4 g_{k}^{2} \int_{0}^{t} \int_{0}^{t}\left(\xi_{k}^{x}\left(\tau_{1}\right)+\xi_{k}^{y}\left(\tau_{1}\right)\right)\left(\xi_{k}^{x}\left(\tau_{2}\right)+\xi_{k}^{y}\left(\tau_{2}\right)\right) d \tau_{2} d \tau_{1}\right]\right)
\end{aligned}
$$


from which we have

$$
\mathbb{E}\left[\exp \left(\imath \varphi_{k}(t)\right)\right]_{\varphi_{k}}=\exp \left(-2 g_{k}^{2} \int_{0}^{t} \int_{0}^{t}\left(\begin{array}{c}
\mathbb{E}\left[\xi_{k}^{x}\left(\tau_{1}\right) \xi_{k}^{x}\left(\tau_{2}\right)\right]+\mathbb{E}\left[\xi_{k}^{y}\left(\tau_{1}\right) \xi_{k}^{y}\left(\tau_{2}\right)\right]+ \\
+\mathbb{E}\left[\xi_{k}^{x}\left(\tau_{1}\right) \xi_{k}^{y}\left(\tau_{2}\right)\right]+\mathbb{E}\left[\xi_{k}^{y}\left(\tau_{1}\right) \xi_{k}^{x}\left(\tau_{2}\right)\right]
\end{array}\right) d \tau_{1} d \tau_{2}\right)
$$

After straightforward calculations, the characteristic function of the noise phase $\varphi_{k}(t)$ in its more explicit form reads:

$$
\mathbb{E}\left[\exp \left(\imath \varphi_{k}(t)\right)\right]_{\varphi_{k}}=\exp \left(-2 g_{k}^{2} \int_{0}^{t} \int_{0}^{t} \mathrm{~K}\left(\tau_{1}, \tau_{2}\right) \cos \left[\omega_{k}\left(\tau_{1}-\tau_{2}\right)\right] d \tau_{1} d \tau_{2}\right) .
$$

Finally, it is easy to see that

$$
\mathbb{E}\left[\exp \left( \pm \imath m \varphi_{k}(t)\right)\right]_{\varphi_{k}}=\exp \left(-2\left(m g_{k}\right)^{2} \chi(t)\right) \equiv \beta_{m}(t)
$$

with

$$
\chi(t)=\int_{0}^{t} \int_{0}^{t} \mathrm{~K}\left(\tau_{1}, \tau_{2}\right) \cos \left[\omega_{k}\left(\tau_{1}-\tau_{2}\right)\right] d \tau_{1} d \tau_{2}
$$

Therefore, the averaged terms of the form $\mathbb{E}\left[\cos \left(m \varphi_{k}(t)\right)\right]_{\varphi_{k}}$ and $\mathbb{E}\left[\sin \left(m \varphi_{k}(t)\right)\right]_{\varphi_{k}}$ can be easily evaluated as follows:

$$
\left\{\begin{array}{l}
\mathbb{E}\left[\cos \left(m \varphi_{k}(t)\right)\right]_{\varphi_{k}}=\mathbb{E}\left[\exp \left( \pm \imath m \varphi_{k}(t)\right)\right]_{\varphi_{k}}=\beta_{m}(t) \\
\mathbb{E}\left[\sin \left(m \varphi_{k}(t)\right)\right]_{\varphi_{k}}=0
\end{array}\right.
$$

As we have already mentioned in the introduction, in this work, we focus on two CSFs with zero average the first with OU autocorrelation function the second with $\mathrm{G}$ autocorrelation function. The OU and G autocorrelation function are given by:

$$
\mathrm{K}_{O U}\left(\tau_{1}, \tau_{2}\right)=\frac{\Gamma}{2 \tau_{c}} \exp \left(-\frac{\left|\tau_{1}-\tau_{2}\right|}{\tau_{c}}\right)
$$

and

$$
\mathrm{K}_{G}\left(\tau_{1}, \tau_{2}\right)=\frac{\Gamma}{\tau_{c} \sqrt{\pi}} \exp \left(-\frac{\left(\tau_{1}-\tau_{2}\right)^{2}}{\tau_{c}^{2}}\right)
$$

The $\chi(t)$ functions associated to these autocorrelations functions are obtained from Eq. (22) and they reads:

$$
\chi_{O U}(t)=\frac{\Gamma}{\left[1+\left(\omega_{k} \tau_{c}\right)^{2}\right]^{2}}\left(\tau_{c} \exp \left(-\frac{t}{\tau_{c}}\right)\left[\left(1-\left(\omega_{k} \tau_{c}\right)^{2}\right) \cos \omega_{k} t-2 \omega_{k} \tau_{c} \sin \omega_{k} t\right]+t-\tau_{c}+\left(t+\tau_{c}\right)\left(\omega_{k} \tau_{c}\right)^{2}\right)
$$

and

$$
\begin{aligned}
\chi_{G}(t) & =\frac{\Gamma}{4} \exp \left(-\frac{\left(\omega_{k} \tau_{c}\right)^{2}}{4}\right)\left[\left(\imath \omega_{k} \tau_{c}^{2}-2 t\right) \operatorname{Erf}\left(\frac{\imath \omega_{k} \tau_{c}^{2}-2 t}{2 \tau_{c}}\right)+\left(\imath \omega_{k} \tau_{c}^{2}+2 t\right) \operatorname{Erf}\left(\frac{\imath \omega_{k} \tau_{c}^{2}+2 t}{2 \tau_{c}}\right)\right]+ \\
& +\frac{\Gamma \tau_{c}}{2 \sqrt{\pi}}\left[\exp \left(\frac{t\left(\imath \omega_{k} \tau_{c}^{2}-t\right)}{\tau_{c}^{2}}\right)+\exp \left(-\frac{t\left(\imath \omega_{k} \tau_{c}^{2}+t\right)}{\tau_{c}^{2}}\right)-\imath \omega_{k} \tau_{c} \sqrt{\pi} \exp \left(-\frac{\left(\omega \tau_{c}\right)^{2}}{4}\right) \operatorname{Erf}\left(\frac{\imath \omega_{k} \tau_{c}}{2}\right)-2\right]
\end{aligned}
$$

Note that the parameter $\tau_{c}$ is the characteristic time of the environment; that is, the time after which the environment correlations cease to be meaningful [1]. $\Gamma$ is the damping rate.

As pointed out in the introduction, we will employ the evolution of tripartite negativity to quantify the dynamics of entanglement of the three-qubit system. In fact, the tripartite negativity which arises from the positive partial transpose (PPT) criterion is defined as [41]

$$
\mathcal{N}^{(3)}(\rho)=\sqrt[3]{\mathcal{N}_{1-23} \mathcal{N}_{2-13} \mathcal{N}_{3-12}}
$$

It can be seen from this definition that the tripartite negativity is nothing else than the geometric average over all the possible bipartition of the tripartite system. $\mathcal{N}_{\ell-m n}=\sum_{\imath}\left|\lambda_{\imath}\left(\rho^{T_{\ell}}\right)\right|-1$ ( with $\ell \in\{1,2,3\}$ and $m n \in\{12,13,23\}$ ) stands for negativity between the bipartition $\ell-m n$ with one qubit ( $\ell$ one) in one block and the remaining two qubits $\left(m\right.$ and $n$ ) in another block. Note that $\rho^{T_{\ell}}$ is the partial transpose of the total density matrix $\rho$ with respect to qubit $\ell$ and $\lambda_{\imath}\left(\rho^{T_{\ell}}\right)$ are its eigenvalues. 


\section{DECOHERENCE PROCESS, TRANSITION AND SURVIVAL PROBABILITY}

Here, we are going to investigate the decoherence process induces by the classical environment portrayed by a Gaussian distributed stochastic field with $\mathrm{OU}$ or $\mathrm{G}$ distribution. To this aim, we concentrate on the decoherence factor (DF) $\beta_{m}(t)$ and explore it environment-parameters dependences in detail. In Fig. 1, we plot the time evolution of the DF $\beta_{2}(t)$ for different values of the parameters $\tau_{c}$ and $\omega_{k} \equiv \omega$ in the case of OU and G noise. It is observe both in the case of OU and $\mathrm{G}$ noise that $\beta_{2}(t)$ is an oscillating function whose amplitude decreases as the time evolves and finally goes to zero at the end of the evolution process (i.e., $\left.\beta_{2}(\infty)=0\right)$. Therefore, we can conclude that at the end of the decoherence process; both noises could be fatal to the quantum properties of any system suffering them. On the hand, we observe that the parameters $\tau_{c}$ and $\omega$ play a significant role in affecting the DP.
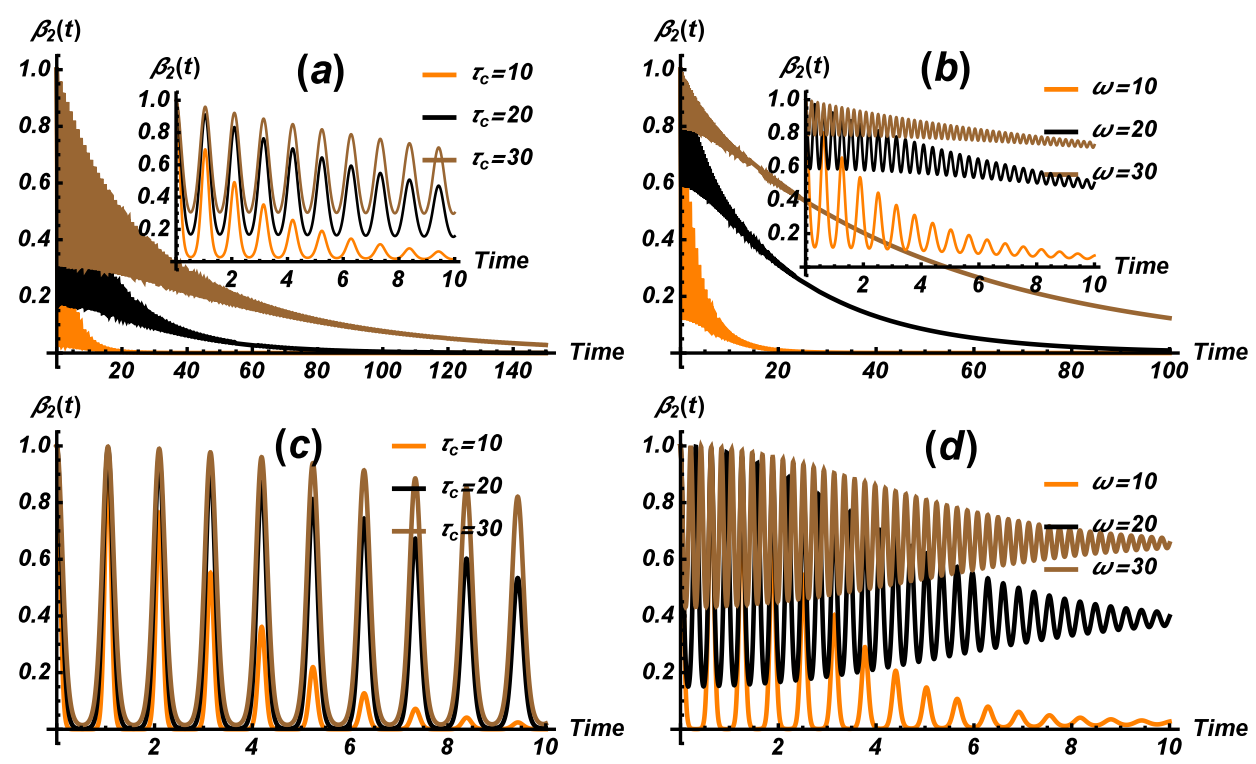

FIG. 1. Upper panel: time evolution of the decoherence factor $\beta_{2}(t)$ for different values of the parameters $\tau_{c}($ with, $\Gamma=5$, $g=4$ and $\omega=6$ ) and $\omega$ (with, $\Gamma=5, g=4$ and $\tau_{c}=6$ ) in the case OU noise. Lower panel: same as in the upper panel but rather in the case $\mathrm{G}$ noise.

In point of fact, we can easily see that decreasing the intensity of the correlations time or the central frequency of the environment only strengthens the detrimental effects of decoherence. That is, the smaller the correlations time or the central frequency of the environment is, the faster is the decoherence process. Moreover, from the explicit expression of decoherence factor $\beta_{m}(t)$ show in Eq. (21), it is easy to see that increasing the parameter $m$ or the system-environment interaction strength (i.e., $g_{k} \equiv g$ ) will also only strengthens the decoherence process. It is instructive to note that in the case of $\mathrm{G}$ noise, they are optimal parameters for which the DF is no longer equal to zero at the end the evolution process (i.e., $\beta_{2}(\infty) \neq 0$ ). These optimal parameters are given as follow: $\Gamma=g=1$, $\tau_{c} \geq 1$ and $\omega \geq 1$. With appropriate choice of these optimal parameters, the DP can even be completely suppressed that is, the DF factor tends to unity after a given instant of time. Therefore, theses optimal parameters can be used to efficiently control quantum resources like entanglement in a system subjected to $\mathrm{G}$ noise. To show this feature, we have plot in Fig. 2 the time evolution of the DF for different optimal values of the parameters $\tau_{c}$ and $\omega$.
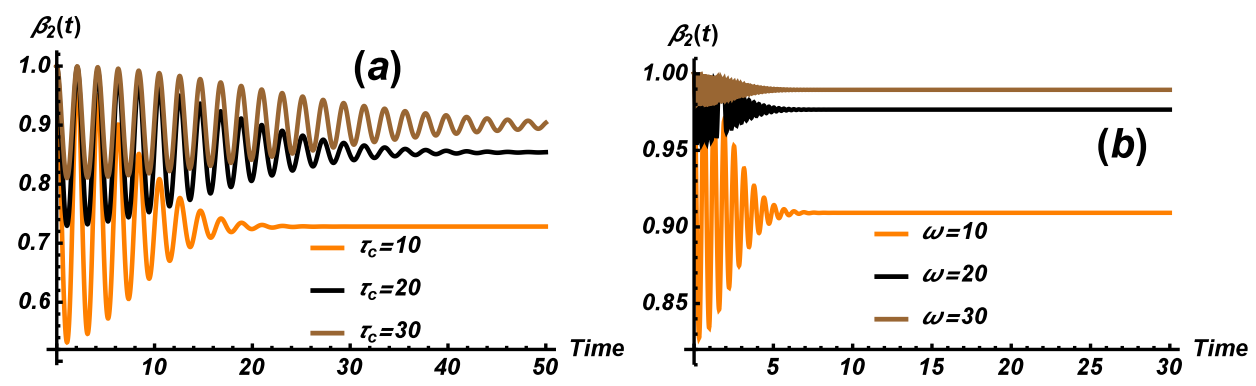

FIG. 2. Evolution of the decoherence factor $\beta_{2}(t)$ for different optimal values of the parameters $\tau_{c}$ and $\omega$ obtained in the case of $\mathrm{G}$ noise with the following set of optimal parameters: (a): $\Gamma=g=1, \omega=3$ and (b): $\Gamma=g=1, \tau_{c}=3$.

We can see in both panels that the DP at the beginning of the evolution is accompanied with rapid damped 
oscillations and then stabilizes at a given instant of time until the end of the evolution process. However, the time domain after which the freezing of the DF occurs depends upon the choosing optimal value of the parameters $\omega$ and $\tau_{c}$. Indeed, it can be seen that the time after which the DF becomes stables increases with the increasing of the optimal values of the aforementioned parameters. It is important to note that the higher is the saturation value of the DF, the less strong is the DP. Therefore, we can effectively suppress the detrimental effect of the G noise by adjusting the noise parameters.

Let us recall that in the physical model under investigation the qubits have non-degenerated energy levels. However, they can also be effectively treated as if their energy levels are degenerated by assuming that the spectrum of external environment holds frequency that are larger than the natural frequency of each of them. This means that under certain conditions as those assuming in this work, the two qubit models (degenerated and non-degenerated) are the same as far as the dynamics is concerned. This leads to the computation of the survival and transition probability in order to see if the transition between two degenerated states is possible. Once the average calculations of the expressions of the survival and transition probability given in Eqs. (10) and (11) are performed, we obtain:

$$
\mathrm{P}_{s}(t)=\frac{1}{2}\left(1+\beta_{2}(t)\right) \text { and } \mathrm{P}_{t r}(t)=\frac{1}{2}\left(1-\beta_{2}(t)\right)=1-\mathrm{P}_{S}(t)
$$

As we have already mentioned, we can see that for isolated degenerated qubit, the transition between the two degenerated states is not possible. In fact, in the absence of noise that is, at $t=0$, the $\mathrm{DF} \beta_{2}(t)$ tends to one and as a consequence, the survival probability takes the value 1. It can also be seen from Eq. (29) that the maximum probability to have a transition from the initial prepared state of the qubit to the other state due the noisy environment is $1 / 2$. In Fig. 3, we have plot the time evolution of these probabilities when the degenerated qubit is affected by the $\mathrm{OU}(\mathrm{a})$ and $\mathrm{G}(\mathrm{b})$ noise.
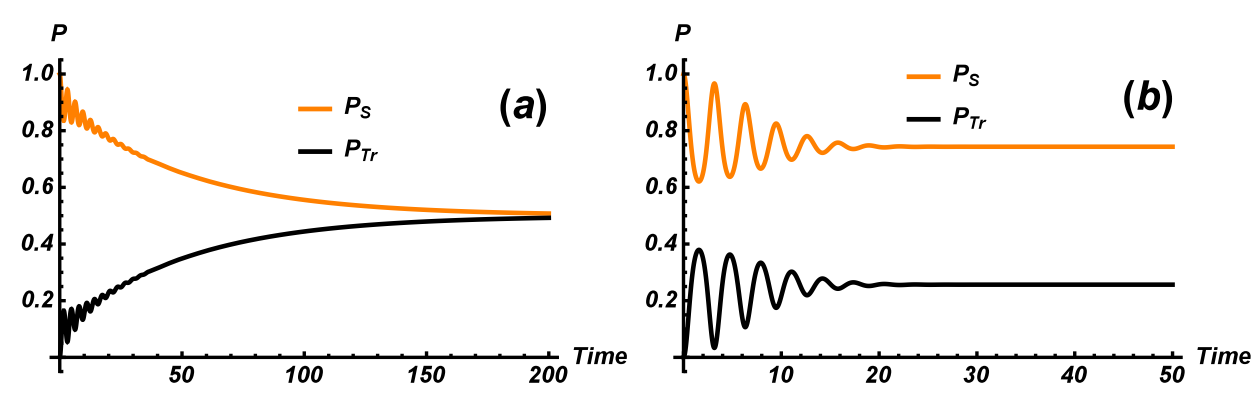

FIG. 3. Time evolution of the survival and transition probability in case of OU (a) and G (b) noise, obtained with the following optimal parameters: $\Gamma=g=1, \omega=2$ and $\tau_{c}=10$

This figure shows how the coupling of the degenerated qubit to its external environment influences the transition/survival probability. It can be observe that the noisy environment can induces transition between the two degenerated states of the qubit. In fact, the figure shows that due to the external environment, the qubit, which was initially localized in one of its degenerated state, evolves to split in both states. However, a complete transfer of the qubit from its initial localized state to the other state is not achieved at the end of the evolution process. In the case of OU noise, we find that the qubit is equally divided in both states at the end of the evolution meanwhile is it not the case for $\mathrm{G}$ noise. This can be explained by noticing that, in the case of $\mathrm{G}$ noise, the optimal parameters can permit to the qubit to adapt to changes imposed by the noise. Therefore, for some particular choice of the optimal parameters, the qubit will then end the evolution in its initial localized state.

\section{ENTANGLEMENT DYNAMICS}

In this section, the analytical as well numerical results for aforementioned qubit-noise coupling (QNC) configurations namely the IEs, MEs and CE are presented. For each QNC configuration, we investigate the situations where the qubits are initially prepared both in the GHZ and W state and coupled to a CSF with either OU or G autocorrelation function. 


\section{A. Independent environments (IEs)}

In this QNC configuration, the evolutions of the system obtained after performing the calculations from Eq. (13) read:

$$
\begin{aligned}
\rho_{G H Z}^{I E s}(t) & =\frac{q\left(3 \beta_{2}^{2}(t)+1\right)}{8}(|000\rangle\langle 111|+| 111\rangle\langle 000|)+\frac{3 q \beta_{2}^{2}(t)+1}{8}(|000\rangle\langle 000|+| 111\rangle\langle 111|)- \\
& -\frac{q \beta_{2}^{2}(t)-1}{8}(|001\rangle\langle 001|+| 010\rangle\langle 010|+| 011\rangle\langle 011|+| 100\rangle\langle 100|+| 101\rangle\langle 101|+| 110\rangle\langle 110|)- \\
& -\frac{q\left(\beta_{2}^{2}(t)-1\right)}{8}(|001\rangle\langle 110|+| 010\rangle\langle 101|+| 011\rangle\langle 100|+| 110\rangle\langle 001|+| 101\rangle\langle 010|+| 100\rangle\langle 011|)
\end{aligned}
$$

and

$$
\begin{aligned}
\rho_{W}^{I E s}(t) & =\frac{\mathcal{P}(t)}{8}(|001\rangle\langle 001|+| 010\rangle\langle 010|+| 100\rangle\langle 100|)+ \\
& +\frac{\mathcal{J}(t)}{12}([|001\rangle+|010\rangle+|100\rangle]\langle 111|+| 111\rangle[\langle 001|+\langle 010|+\langle 100|])+ \\
& +\frac{\mathcal{G}(t)}{12}([|011\rangle+|101\rangle+|110\rangle]\langle 000|+| 000\rangle[\langle 011|+\langle 101|+\langle 110|])+ \\
& +\frac{\mathcal{F}(t)}{12}(|001\rangle[\langle 010|+\langle 100|]+\mid 100\rangle[\langle 010|+\langle 001|]+\mid 010\rangle[\langle 001|+\langle 100|])+ \\
& +\frac{\mathcal{D}(t)}{12}(|011\rangle[\langle 010|+\langle 101|]+\mid 101\rangle[\langle 011|+\langle 110|]+\mid 110\rangle[\langle 011|+\langle 101|])+ \\
& +\frac{\mathcal{L}(t)}{8}|000\rangle\left\langle 000\left|+\frac{\mathcal{K}(t)}{8}\right| 111\right\rangle\langle 111|+\frac{\mathcal{Y}(t)}{8}(|011\rangle\langle 011|+| 101\rangle\langle 110|+| 110\rangle\langle 110|)
\end{aligned}
$$

with

$$
\begin{aligned}
& \mathcal{L}(t)=q\left[\left(1+\beta_{2}(t)\right)^{2}\left(1-\beta_{2}(t)\right)-1\right]+1, \mathcal{K}(t)=q\left[\left(1-\beta_{2}(t)\right)^{2}\left(1+\beta_{2}(t)\right)-1\right]+1, \\
& \mathcal{J}(t)=q\left(1-\beta_{2}(t)\right)^{2}\left(1+\beta_{2}(t)\right), \mathcal{P}(t)=\frac{q}{3}\left[\left(1+\beta_{2}(t)\right)\left[\left(1+\beta_{2}(t)\right)^{2}+2\left(1-\beta_{2}(t)\right)^{2}\right]-3\right]+1, \\
& \mathcal{P}(t)=\frac{q}{3}\left[\left(1+\beta_{2}(t)\right)\left[-3\left(1+\beta_{2}(t)\right)^{2}-2\left(1-5 \beta_{2}(t)\right)^{2}\right]+5\right]+1, \mathcal{F}(t)=q\left(1+\beta_{2}(t)\right)\left(1+\beta_{2}^{2}(t)\right), \\
& \mathcal{D}(t)=q\left(1-\beta_{2}(t)\right)\left(1+\beta_{2}^{2}(t)\right), \text { and } \mathcal{G}(t)=q\left(1-\beta_{2}(t)\right)\left(1+\beta_{2}(t)\right)^{2} .
\end{aligned}
$$

Note that in the above expressions the time dependent function $\beta_{2}(t)$ is the decoherence factor whose explicit expression is given in Eq.(21) and depends on the specific choice of the CSF via the function $\chi(t)$ (Eqs. (26) and (27)). With these density matrices in our hand, we can then investigate the dynamics of entanglement by employing the tripartite negativity as given in Eq. (28). However, it is important to note that because of lack of compact analytical results, only numerical ones are presented.

In Figs. 4 and 5, we report the evolution of the tripartite negativity for different optimal values of the parameters $\omega$ (central frequency of the CSF), and $\tau_{c}$ (characteristic time of the CSF) as a function of time both for the initial GHZ (upper panel) and W (lower panel) state in the case of OU and G noise respectively. As first observation, we can see that the entanglement exhibits almost the same qualitative behaviors both for GHZ and W initial states, regardless of the central frequency and characteristic time of the noisy environment. However, one can also observe both in Figs. 4 and 5 that during the evolution, the residual amount of entanglement in the GHZ state is always larger than the one in the $\mathrm{W}$ state, demonstrating that the $\mathrm{W}$ state is more fragile under this model of decoherence in the IEs configuration than the GHZ state. It is also observe that whatever the kind of noise considered (OU or G noise), entanglement shows damped oscillation as the time evolves. However, the total suppression of entanglement is unavoidable when the system is coupled to the OU noise meanwhile in the case of G noise, optimal parameters allows the system to adapt to changes imposed by the external noise and thereby, the system is not completely disentangled. In point of fact, we observed in both figures that entanglement decays with damped oscillations and finally disappears asymptotically in the limit of long time (Fig. (4) or abruptly freezes to a particular value at a given instant of time (Fig. 5). We can see in Fig. 4 that, the time domain after which the complete disentanglement of the system occurs increases with the increasing of the parameters $\tau_{c}$ and $\omega$. On the other hand, we can see in Fig. [5] that the time span after which the residual amount of entanglement in the system freezes increases also with both parameters. Those results are not surprising because it is consistent with those obtained during the investigation of the decoherence process by means of the time evolution of the DF. It is worth noting that the increases of the survival time or freezing amount of entanglement with the parameter $\tau_{c}$ is meaningful but not surprising because it is 

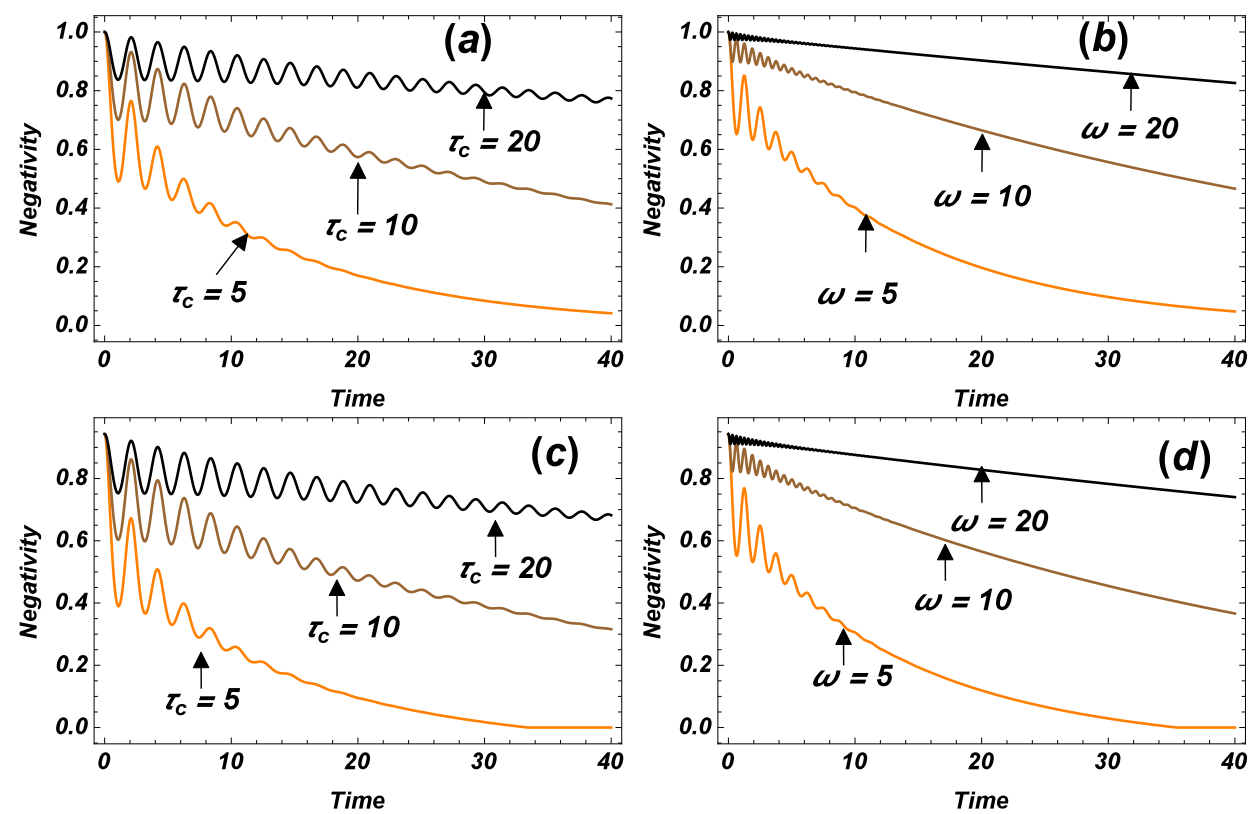

FIG. 4. Upper panel: time evolution of the tripartite negativity for different values of $\tau_{c}$ (a), with $(g=\Gamma=q=1$ and $\omega=3)$ and $\omega(\mathrm{b})$, with $\left(g=\Gamma=q=1\right.$ and $\left.\tau_{c}=3\right)$, when the qubits initially prepared in the GHZ state are coupled to a CSF with OU distribution in independent environments. Lower panel: same as in the upper one but when the qubits are prepared in the w state.
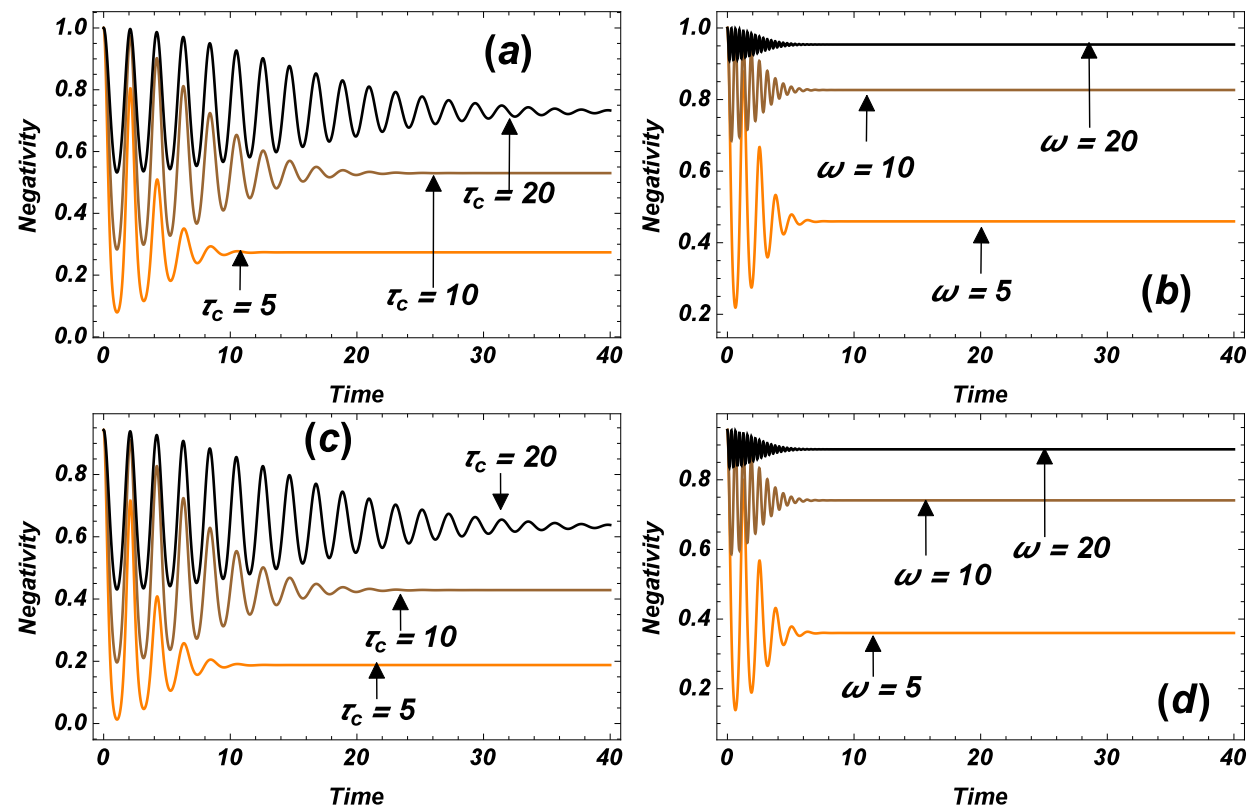

FIG. 5. Upper panel: time evolution of the tripartite negativity for different values of $\tau_{c}(\mathrm{a})$, with $(g=\Gamma=q=1$ and $\omega=3)$ and $\omega(\mathrm{b})$, with $\left(g=\Gamma=q=1\right.$ and $\left.\tau_{c}=3\right)$, when the qubits initially prepared in the GHZ state are coupled to a CSF with Gaussian distribution in independent environments. Lower panel: same as in the upper one but when the qubits are prepared in the $\mathrm{w}$ state.

well known that the autocorrelation time $\tau_{c}$ measures the time after which the environment correlations cease to be meaningful or can also be interpreted as the amount of time the environment can store information for [1]. As such, if $\tau_{c}$ is short, the memory of the environment is also short and as a consequence, the amount of information back and forth from the environment to the system and vice versa due to the memory effects of the environment is not significant. Although quantitatively different, the behaviors of entanglement obtained for different optimal values of the parameter $\omega$, is almost qualitatively similar to those obtained for different optimal values of the parameter $\tau_{c}$, demonstrating that both parameters play the same role in affecting the evolution of entanglement. Overall, it is observe that the decoherence induces by OU noise is more fatal to the survival of entanglement than the one induces by $\mathrm{G}$ noise and that the detrimental effects of both noises can be softened under certain particular environment's 
parameters.

\section{B. Mixed environments (MEs)}

In the previous subsection we have investigated the situation where the qubits are locally coupled the noise (IEs). Now let's look for the situation where they are coupled in MEs. After performing the calculations, the resulting time-evolved density matrix for initial GHZ-type states can be written in three qubit basis as:

$$
\begin{aligned}
\rho_{G H Z}^{M E S}(t) & =\mathcal{F}(t)(|010\rangle\langle 010|+| 011\rangle\langle 011|+| 100\rangle\langle 100|)+ \\
& +\mathcal{Q}(t)(|111\rangle\langle 111|+| 000\rangle\langle 000|)+\mathcal{S}(t)(|001\rangle\langle 001|+| 110\rangle\langle 110|)+ \\
& +\mathcal{K}(t)(|000\rangle\langle 111|+| 111\rangle\langle 000|)+\mathcal{J}(t)(|001\rangle\langle 110|+| 110\rangle\langle 001|)+ \\
& +\mathcal{R}(t)\left(\begin{array}{l}
(|111\rangle+|000\rangle)(\langle 001|+\langle 110|)+(|001\rangle+|110\rangle)(\langle 000|+\langle 111|)- \\
-|010\rangle(\langle 011|+\langle 100|+\langle 101|)-\mid 011\rangle(\langle 010|+\langle 100|+\langle 101|)- \\
-|100\rangle(\langle 010|+\langle 011|+\langle 101|)-\mid 101\rangle(\langle 010|+\langle 100|+\langle 011|)
\end{array}\right)
\end{aligned}
$$

with

$$
\begin{aligned}
& \mathcal{Q}(t)=\frac{1}{8}\left(\frac{q}{2}\left(1+\beta_{4}(t)+4 \beta_{2}^{2}(t)\right)+1\right), \mathcal{S}(t)=\frac{1}{8}\left(\frac{q}{2}\left(1+\beta_{4}(t)-4 \beta_{2}^{2}(t)\right)+1\right), \mathcal{R}(t)=\frac{q}{16}\left(\beta_{4}(t)-1\right), \\
& \mathcal{F}(t)=\frac{1}{8}\left(-\frac{q}{2}\left(1+\beta_{4}(t)\right)+1\right), \mathcal{K}(t)=\frac{q}{16}\left(3+\beta_{4}(t)+4 \beta_{2}^{2}(t)\right) \text { and } \mathcal{J}(t)=\frac{q}{16}\left(3+\beta_{4}(t)-4 \beta_{2}^{2}(t)\right)
\end{aligned}
$$

For initial W-type states, the time-evolved density matrix takes can be written in the three qubits basis in its more explicit form as:

$$
\begin{aligned}
\rho_{W}^{M E s} & =\mathcal{Q}(t)|000\rangle\langle 000|+\mathcal{R}(t)| 111\rangle\langle 111|+\mathcal{T}(t)| 001\rangle\langle 001|+\mathcal{J}(t)| 110\rangle\langle 110|+ \\
& +\mathcal{G}(t)(|011\rangle\langle 011|+| 101\rangle\langle 101|)+\mathcal{H}(t)(|010\rangle\langle 010|+| 100\rangle\langle 100|)+ \\
& +\mathcal{I}(t)[(|011\rangle+|101\rangle)\langle 111|+| 000\rangle(\langle 011|+\langle 101|)]+\mathcal{D}(t)(|000\rangle\langle 110|+| 110\rangle\langle 000|)+ \\
& +\mathcal{W}(t)[(|010\rangle+|100\rangle)\langle 001|+| 001\rangle(\langle 010|+\langle 100|)]+\mathcal{Z}(t)(|001\rangle\langle 111|+| 111\rangle\langle 001|)+ \\
& +\mathcal{L}(t)[(|010\rangle+|100\rangle)\langle 111|+| 111\rangle(\langle 010|+\langle 100|)]+\mathcal{S}(t)(|011\rangle\langle 101|+| 101\rangle\langle 011|)+ \\
& +\mathcal{P}(t)[(|011\rangle+|101\rangle)\langle 110|+| 110\rangle(\langle 011|+\langle 101|)]+\mathcal{E}(t)(|010\rangle\langle 100|+| 100\rangle\langle 010|)
\end{aligned}
$$

with

$$
\begin{aligned}
& \mathcal{Q}(t)=\frac{q}{48}+\frac{1}{8}+q\left(-\frac{\beta_{2}^{2}(t)}{12}+\frac{5 \beta_{2}(t)}{48}-\frac{\beta_{4}(t)}{16}-\frac{10 \beta_{2}(t) \beta_{4}(t)}{96}\right), \mathcal{W}(t)=\frac{q}{12}\left(\beta_{2}^{2}(t)+\beta_{2}(t)+\beta_{2}(t) \beta_{4}(t)+1\right), \\
& \mathcal{R}(t)=\frac{q}{48}+\frac{1}{8}+q\left(-\frac{\beta_{2}^{2}(t)}{12}-\frac{5 \beta_{2}(t)}{48}-\frac{\beta_{4}(t)}{16}+\frac{10 \beta_{2}(t) \beta_{4}(t)}{96}\right), \mathcal{I}(t)=-\frac{q}{12}\left(\beta_{2}^{2}(t)-\beta_{2}(t)+\beta_{2}(t) \beta_{4}(t)-1\right), \\
& \mathcal{T}(t)=\frac{q}{48}+\frac{1}{8}+q\left(\frac{\beta_{2}^{2}(t)}{12}+\frac{\beta_{2}(t)}{16}-\frac{\beta_{4}(t)}{16}+\frac{10 \beta_{2}(t) \beta_{4}(t)}{96}\right), \mathcal{L}(t)=-\frac{q}{12}\left(\beta_{2}^{2}(t)-\beta_{2}(t)-\beta_{2}(t) \beta_{4}(t)-1\right), \\
& \mathcal{J}(t)=\frac{q}{48}+\frac{1}{8}+q\left(\frac{\beta_{2}^{2}(t)}{12}-\frac{\beta_{2}(t)}{16}-\frac{\beta_{4}(t)}{16}-\frac{10 \beta_{2}(t) \beta_{4}(t)}{96}\right), \mathcal{P}(t)=-\frac{q}{12}\left(-\beta_{2}^{2}(t)+\beta_{2}(t)+\beta_{2}(t) \beta_{4}(t)-1\right), \\
& \mathcal{H}(t)=-\frac{q}{48}+\frac{1}{8}+q\left(\frac{\beta_{2}(t)}{16}+\frac{\beta_{4}(t)}{16}+\frac{10 \beta_{2}(t) \beta_{4}(t)}{96}\right), \mathcal{D}(t)=-\frac{q}{96}\left(6 \beta_{4}(t)-10 \beta_{2}(t)+10 \beta_{2}(t) \beta_{4}(t)-6\right), \\
& \mathcal{G}(t)=-\frac{q}{48}+\frac{1}{8}+q\left(-\frac{\beta_{2}(t)}{16}+\frac{\beta_{4}(t)}{16}-\frac{10 \beta_{2}(t) \beta_{4}(t)}{96}\right), \mathcal{D}(t)=-\frac{q}{96}\left(6 \beta_{4}(t)+10 \beta_{2}(t)-10 \beta_{2}(t) \beta_{4}(t)-6\right), \\
& \mathcal{S}(t)=\frac{q}{96}\left(6 \beta_{4}(t)-6 \beta_{2}(t)-10 \beta_{2}(t) \beta_{4}(t)+10\right) \text { and } \mathcal{E}(t)=\frac{q}{96}\left(6 \beta_{4}(t)+6 \beta_{2}(t)+10 \beta_{2}(t) \beta_{4}(t)+10\right) .
\end{aligned}
$$

As we have already pointed out, the analytical results of entanglement are very large. Therefore, we will resort only to numerical results for studying the dynamics of entanglement for this QNC configuration. In Figs. 6] and 7 we have 

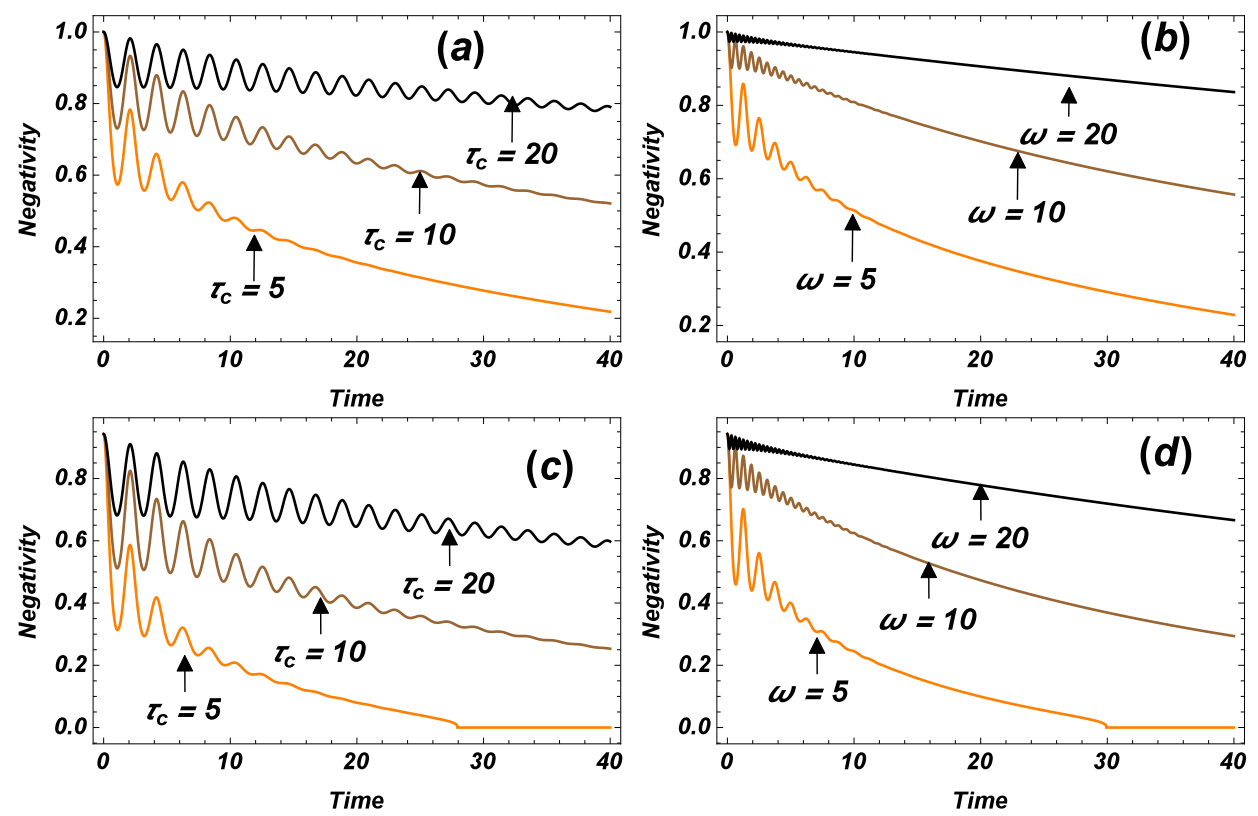

FIG. 6. Upper panel: time evolution of the tripartite negativity for different values of $\tau_{c}$ (a), with $(g=\Gamma=q=1$ and $\omega=3)$ and $\omega(\mathrm{b})$, with $\left(g=\Gamma=q=1\right.$ and $\left.\tau_{c}=3\right)$, when the qubits initially prepared in the GHZ state are coupled to a CSF with OU distribution in mixed environments. Lower panel: same as in the upper one but when the qubits are prepared in the w state.
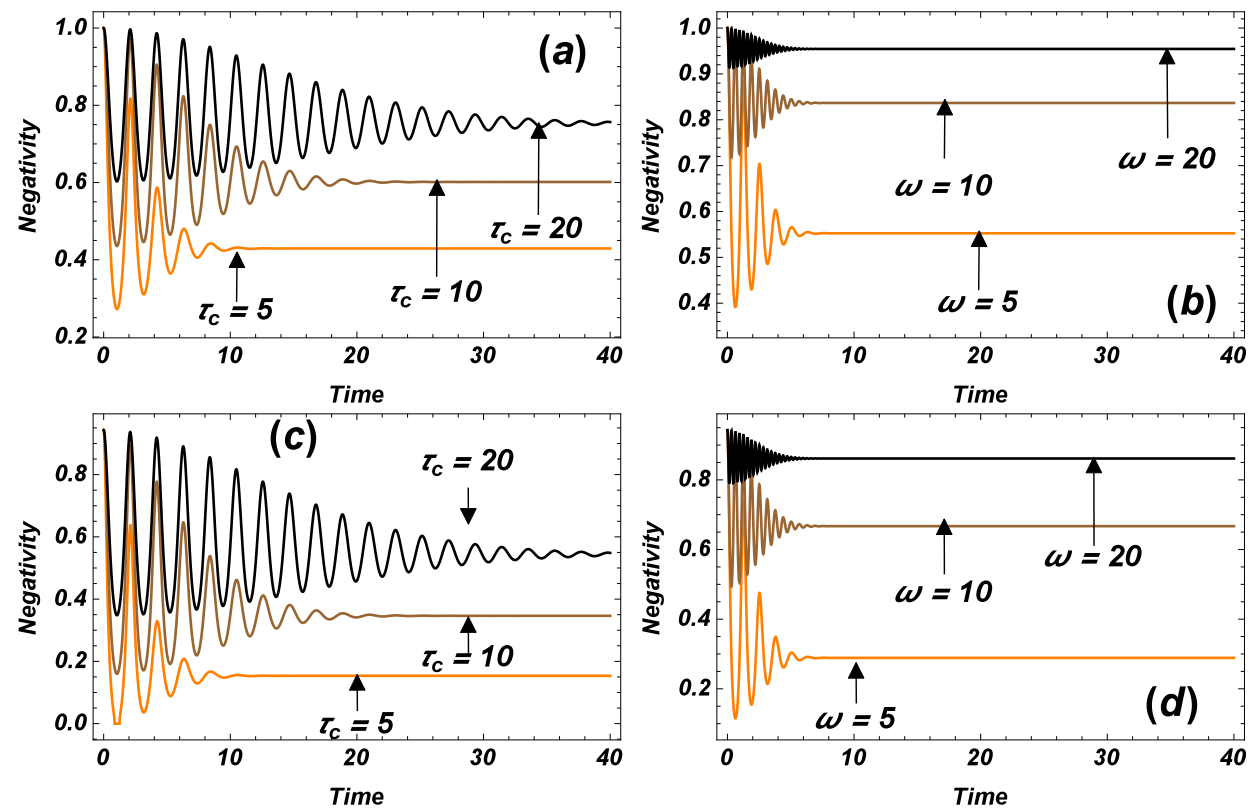

FIG. 7. Upper panel: time evolution of the tripartite negativity for different values of $\tau_{c}(\mathrm{a})$, with $(g=\Gamma=q=1$ and $\omega=3)$ and $\omega(\mathrm{b})$, with $\left(g=\Gamma=q=1\right.$ and $\left.\tau_{c}=3\right)$, when the qubits initially prepared in the GHZ state are coupled to a CSF with Gaussian distribution in mixed environments. Lower panel: same as in the upper one but when the qubits are prepared in the w state.

plot the time evolution of entanglement for different values of $\tau_{c}$ and $\omega$ both for the case of OU and G noise and for GHZ and W state respectively. Although quantitatively different, we can see that the behaviors of entanglement as shown in Figs. 6 and 7 are qualitatively similar to those depicted in Figs. 4 and 5 . This clearly demonstrates that the evolution the entanglement strongly depends on the way the qubits are coupled to the external environment. Indeed, a comparison with Figs. 4 and 5 shows that at a given instant of time, the residual amount of entanglement in the system depends on whether the qubits are coupled to the external noise in MEs or in IEs. More precisely, it is observed that whatever is the kind of noise considered, the residual amount of entanglement in the system when it is initially prepared in the GHZ state is always higher when the qubits are coupled to the noise in MEs than in IEs. In other words, when the qubits are initially prepared in GHZ state, the MEs configuration preserves better 
entanglement than the IEs one. However, the opposite situation occurs when the qubits are initially prepared in W state. That is, in W state, the IEs configuration preserves better entanglement among the qubits than the MEs one. Such results clearly demonstrate that the evolution of entanglement in a system of three qubits not only depends on their initial prepared state but also on the way there are coupled to the noise. Another, remarkable aspect which has already pointed out in the case of IEs is that there exist optimal values of the parameters $\tau_{c}$ and $\omega$ for which it is possible to delay or avoid the complete disentanglement of the system. As in the case of IEs, it can be seen in this setup that entanglement survives for a long time when the qubits are initially set in the GHZ state than in the W state, demonstrating once more the robustness in this decoherence model of the GHZ state with respect to the W one.

\section{Common environment (CE)}

In the two previous subsections, the dynamics of entanglement have been investigated in in the situation where the qubits are coupled to the noise in IEs or MEs respectively. Now in this section, we are going to investigated the situation where the qubits are coupled in a CE. In this situation, the density matrix describing the evolution of the system can be written as:

In the GHZ-type states we have

$$
\begin{aligned}
& \rho_{G H Z}^{C E}(t)=\mathcal{Q}(t)(|000\rangle\langle 000|+| 111\rangle\langle 111|)+\mathcal{T}(t)(|000\rangle\langle 111|+| 111\rangle\langle 000|)+ \\
& +\mathcal{R}(t)(|001\rangle\langle 001|+| 010\rangle\langle 010|+| 011\rangle\langle 011|+| 100\rangle\langle 100|+| 101\rangle\langle 101|+| 110\rangle\langle 110|)+ \\
& +\mathcal{J}(t)\left(\begin{array}{l}
-(|001\rangle+|010\rangle+|011\rangle+|100\rangle+|101\rangle+|110\rangle)(\langle 000|+\langle 111|)- \\
-(|000\rangle+|111\rangle)(\langle 001|+\langle 010|+\langle 011|+\langle 100|+\langle 101|+\langle 110|)+ \\
+|001\rangle(\langle 010|+\langle 011|+\langle 100|+\langle 101|+\langle 110|) \mid 010\rangle(\langle 001|+\langle 011|+\langle 100|+\langle 101|+\langle 110|)+ \\
+|011\rangle(\langle 010|+\langle 001|+\langle 100|+\langle 101|+\langle 110|) \mid 100\rangle(\langle 010|+\langle 011|+\langle 001|+\langle 101|+\langle 110|)+ \\
+|101\rangle(\langle 010|+\langle 011|+\langle 100|+\langle 001|+\langle 110|) \mid 110\rangle(\langle 010|+\langle 011|+\langle 100|+\langle 101|+\langle 001|)
\end{array}\right)
\end{aligned}
$$

where

$\mathcal{Q}(t)=\frac{1}{8}\left(\frac{q}{2}\left(\beta_{4}(t)+1\right)+1\right), \mathcal{R}(t)=\frac{1}{8}\left(-\frac{q}{2}\left(\beta_{4}(t)+1\right)+1\right), \mathcal{T}(t)=\frac{q}{16}\left(3 \beta_{4}(t)+5\right)$ and $\mathcal{J}(t)=-\frac{q}{16}\left(3 \beta_{4}(t)-1\right)$

In the case of $\mathrm{W}$-type states we have

$$
\begin{aligned}
\rho_{W}^{C E}(t) & =\mathcal{Q}(t)|000\rangle\langle 000|+\mathcal{R}(t)| 111\rangle\langle 111|+\mathcal{T}(t)(|001\rangle\langle 001|+| 010\rangle\langle 010|+| 100\rangle\langle 100|)+ \\
& +\mathcal{G}(t)(|011\rangle\langle 011|+| 101\rangle\langle 101|+| 110\rangle\langle 110|)+ \\
& +\mathcal{I}(t)[(|001\rangle+|101\rangle+|110\rangle)\langle 000|+| 000\rangle(\langle 011|+\langle 101|+\langle 110|)]+ \\
& +\mathcal{L}(t)[(|010\rangle+|100\rangle+|001\rangle)\langle 111|+| 111\rangle(\langle 100|+\langle 010|+\langle 001|)]+ \\
& +\mathcal{W}(t)[(|010\rangle+|100\rangle)\langle 001|+| 001\rangle(\langle 010|+\langle 100|)+\mid 010\rangle\langle 100|+| 100\rangle\langle 010|]+ \\
& +\mathcal{P}(t)[(|011\rangle+|101\rangle)\langle 110|+| 110\rangle(\langle 011|+\langle 101|)+\mid 011\rangle\langle 101|+| 101\rangle\langle 011|]
\end{aligned}
$$

with

$$
\begin{aligned}
& \mathcal{Q}(t)=\frac{1}{8}+q\left(\frac{1}{16}+\frac{3 \beta_{2}(t)}{32}-\frac{3 \beta_{4}(t)}{16}-\frac{3 \beta_{6}(t)}{32}\right), \mathcal{T}(t)=\frac{1}{8}+q\left(-\frac{1}{48}+\frac{7 \beta_{2}(t)}{96}+\frac{\beta_{4}(t)}{16}+\frac{3 \beta_{6}(t)}{32}\right), \\
& \mathcal{G}(t)=\frac{1}{8}+q\left(-\frac{1}{48}-\frac{7 \beta_{2}(t)}{96}+\frac{\beta_{4}(t)}{16}-\frac{3 \beta_{6}(t)}{32}\right), \mathcal{R}(t)=\frac{1}{8}+q\left(\frac{1}{16}-\frac{3 \beta_{2}(t)}{32}-\frac{3 \beta_{4}(t)}{16}+\frac{3 \beta_{6}(t)}{32}\right), \\
& \mathcal{I}(t)=\frac{q}{96}\left(6+9 \beta_{2}(t)-6 \beta_{4}(t)-9 \beta_{6}(t)\right), \mathcal{W}(t)=\frac{q}{96}\left(10+7 \beta_{2}(t)+6 \beta_{4}(t)+9 \beta_{6}(t)\right), \\
& \mathcal{P}(t)=\frac{q}{96}\left(10-7 \beta_{2}(t)+6 \beta_{4}(t)-9 \beta_{6}(t)\right) \text { and } \mathcal{L}(t)=\frac{q}{96}\left(6-9 \beta_{2}(t)-6 \beta_{4}(t)+9 \beta_{6}(t)\right) .
\end{aligned}
$$



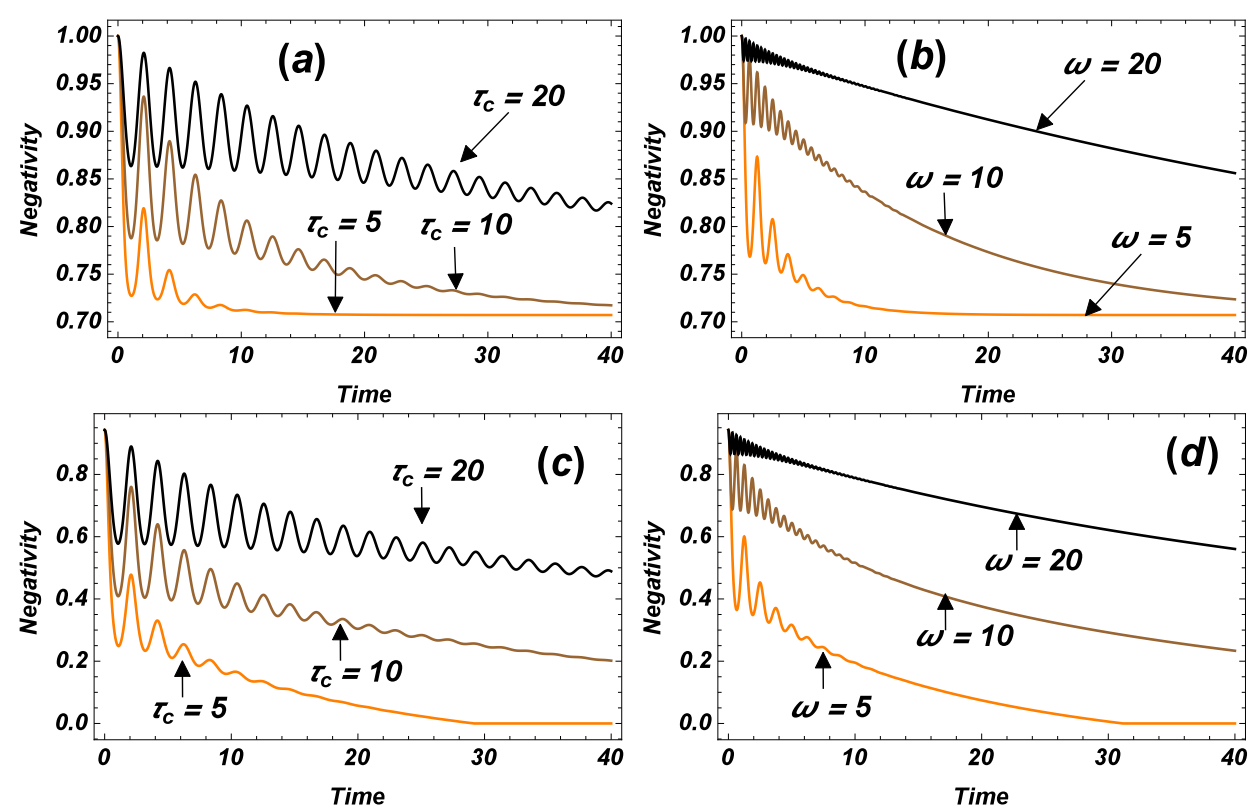

FIG. 8. Upper panel: time evolution of the tripartite negativity for different values of $\tau_{c}(\mathrm{a})$, with $(g=\Gamma=q=1$ and $\omega=3)$ and $\omega(\mathrm{b})$, with $\left(g=\Gamma=q=1\right.$ and $\left.\tau_{c}=3\right)$, when the qubits initially prepared in the GHZ state are coupled to a CSF with OU distribution in a common environment. Lower panel: same as in the upper one but when the qubits are prepared in the w state.
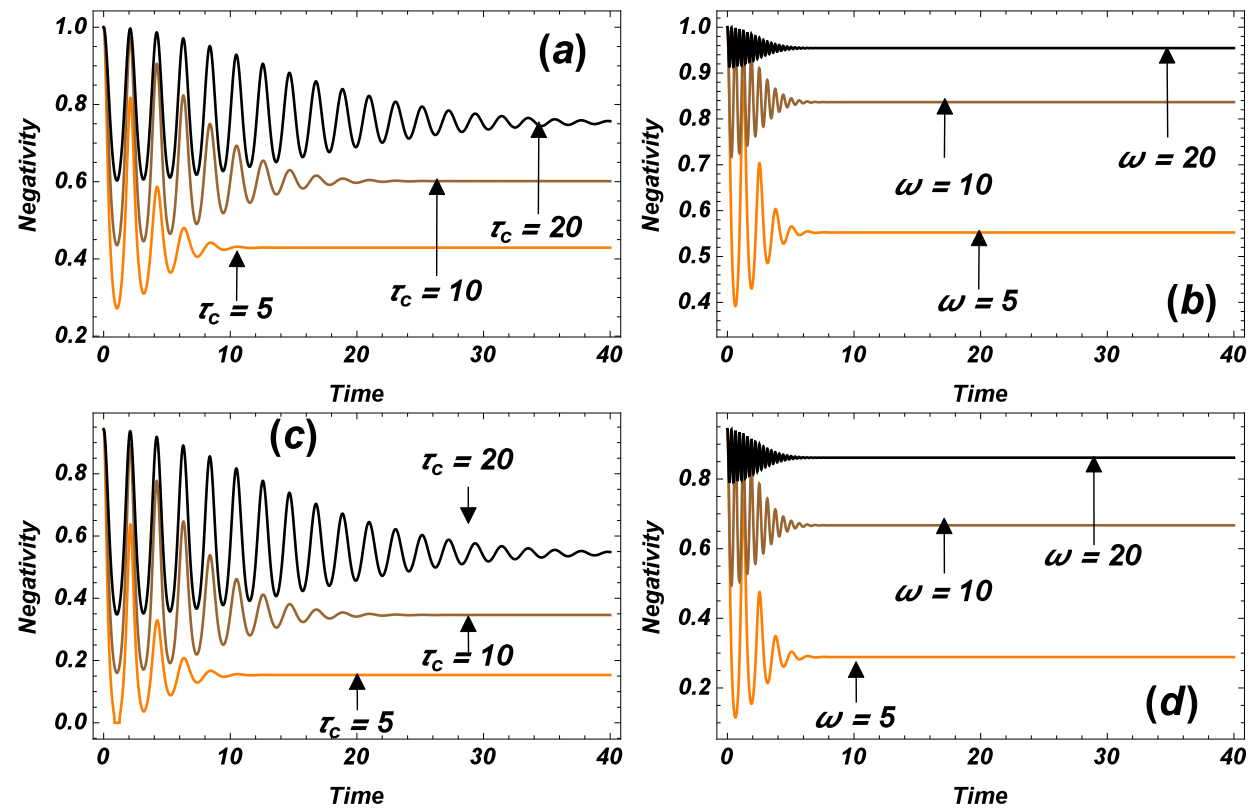

FIG. 9. Upper panel: time evolution of the tripartite negativity for different values of $\tau_{c}$ (a), with $(g=\Gamma=q=1$ and $\omega=3)$ and $\omega(\mathrm{b})$, with $\left(g=\Gamma=q=1\right.$ and $\left.\tau_{c}=3\right)$, when the qubits initially prepared in the GHZ state are coupled to a CSF with Gaussian distribution in a common environment. Lower panel: same as in the upper one but when the qubits are prepared in the w state.

Note that the time dependent functions $\beta_{m}(t)(\mathrm{m}=2,4,6)$ are the so called decoherence factors ant their explicit expressions are given in Eq. 21. One can immediately note that the time-evolved density matrices of the system take different forms depending on whether the qubits are coupled in IEs, MEs or in a CE. Although the analytical expressions of the time evolution of entanglement is not very enlightening, they are not presented because of they cannot be put into a compact forms.

In Figs. 8 and 9, we have plot the time evolution of entanglement (when the qubits are coupled in a CE) for different optimal values of the parameter $\tau_{c}$ and $\omega$ both for the case of GHZ-and W-type initial states. Fig. 8 is for the case OU noise while Fig. 9 is for the case of G noise. It can be observed that even if the entanglement in this QNC configuration shows essentially the same qualitative behaviors as in the case of IEs and MEs configuration, the residual 
amount of entanglement in the system at a given instant of time depends on the QNC configuration considered, that is, the disentanglement of the system is quantitatively different from one configuration to another. Unlike what has been found in the case of IEs and MEs configuration in which entanglement is completely suppressed when the qubits are subjected to the OU noise (regardless of their initial prepared state), we observe that entanglement is indefinitely preserved when the qubits (initially prepared in the GHZ state) are coupled with the OU noise in a CE. This result with those obtained in the previous sections clearly demonstrate that when the qubits are initially prepared in the GHZ state, the CE configuration followed by the MEs configuration preserves better the entanglement or delays the disentanglement of the qubits than the IEs configuration. Such a result is in good agreement with what has been found in Refs. [19, 22]. However, the opposite situation occurs when the qubits are initially set in the W state, that is, when the qubits are prepared in the W state, the CE configuration followed by MEs one hastens the disentanglement of the qubits than the IEs setup. It is worth noting that the long time survival of entanglement as shown in Fig. 8(a) and (b), has been interpreted as a consequence of the direct interaction between the qubits due to their coupling to the same source of noise (common environment) 17]. Another remarkable aspect is that when the qubits are coupled with the OU noise and initially prepared in the GHZ state (Fig. 8 (a) and (b)), the saturation value of entanglement does not depends on the parameter $\tau_{c}$ and $\omega$. In fact, we can see that whatever the value of these parameters are, entanglement abruptly decays with damped oscillations and then freezes to 0.7 for the rest of the time. However, regardless of the kind of noise as well as the initial state considered, the time span after which the entanglement freezes increases with the parameters $\tau_{c}$ and $\omega$.

Overall, the results obtained in this subsection together with those of the two previous subsections clearly show that the main conclusions of the analysis of the entanglement dynamics are almost qualitatively independent on the QNC configuration considered. However, the entanglement dynamics strongly depends both quantitatively and qualitatively on the nature of the CSF used to model the external environment, regardless of the QNC configuration considered.

\section{CONCLUSION}

In this paper, we have investigated the decoherence process induces by a classical environmental noise portrayed by a classical Gaussian distributed stochastic field with either Ornstein-Uhlenbeck autocorrelation function (OU noise) or Gaussian autocorrelation function ( $\mathrm{G}$ noise). We have also investigate in detail the dynamics of entanglement of a system of three noninteracting qubits suffering the detrimental effects of such a decoherence process. Two initial states of the qubits namely, the GHZ- and W-type states have been considered. The qubit-noise coupling has been considered in three different scenarios namely, the common, independent and mixed environment(s). By performing an ensemble average over all the possible realizations of the classical stochastic field, we eliminate the environment's degree of freedoms and obtain the dynamics of the system.

As far as the dynamics of the system is concerned, we found that if the noise spectrum of the classical stochastic field contains frequencies that are larger than the natural frequencies of the qubits, the physical model under investigation (with non-degenerated qubits energy levels) reduces to those of Refs. [17, 19, 22] where qubit energy degeneracy is assumed. Hence, we have computed the transition and survival probability between the two degenerated states of the qubit and found that the qubit will remains in its initial localized state in the absence of noise meanwhile the transition may occurs in the presence of noise.

We found that the decoherence process (DP) strongly depends on the noise considered as well as on the environment parameters namely, its central frequency $(\omega)$ and autocorrelation time $\left(\tau_{c}\right)$. In fact, we found that that decreasing the intensity of $\omega$ and $\tau_{c}$ only strengthens the DP. However, in the case G noise, we found that there are optimal values of the parameters $\omega$ and $\tau_{c}$ for which the DP can be partially or completely suppressed, which thus permits to the system to adapt to changes imposed by the noisy environment.

We found that the OU noise is more fatal to the survival of entanglement than the G noise and that the disentanglement of the system due to the detrimental actions of both noises can be delays (OU noise) or completely suppressed ( $\mathrm{G}$ noise) by suitably choosing the optimal values of the parameters $\omega$ and $\tau_{c}$. In point of fact, we found that a high value of the parameters $\omega$ or $\tau_{c}$ shields the evolution of the system from the detrimental effects of the external noise.

We found that the QNC configuration as well the initial prepared state of the qubits plays an important role towards the preservation of the system's entanglement. In fact, we found that under the physical model considered in this paper, the GHZ state is more robust than the $\mathrm{W}$ state that is, it preserves more entanglement than the $\mathrm{W}$ state. On the other hand, we found that when the qubits are initially set into the GHZ state the CE configuration followed by the MEs configuration delays more the disentanglement of the system than the IEs configuration. However, we found that the situation is completely reversed when the qubits are initially set in the $\mathrm{W}$ state that is, the CE configuration followed by the MEs configuration hastens more the disentanglement of the system than the IEs configuration. 


\section{ACKNOWLEDGMENTS}

This research did not receive any specific grant from funding agencies in the public, commercial, or not-for-profit sectors.

[1] J. Trapani, PhD thesis: Stochastic noise approach to non-Markovian decoherence in continuous variable open quantum systems, Universita degli studi di Milano, (2017).

[2] D.P. DiVincenzo, Fort. Phys. 48, 9 (2000).

[3] C.H. Benneth, et al., Phys. Rev. Lett. 70, 1895 (1993).

[4] X. Li et al., Phys. Rev. Lett. 88, 04790416 (2002).

[5] N. Gisin, et al., Rev. Mod. Phys. 74, 145 (2002).

[6] M.A.C. Rossi, C. Benedetti, M.G.A. Paris, Int. J. Quantum Inf. 12, 1560003 (2014).

[7] C. Benedetti, M.G.A. Paris, Int. J. Quantum Inf. 12, 1461004 (2014).

[8] M.G.A. Paris, Physica A. 413, 256 (2014).

[9] P. Sibani, J. H. Jensen, Stochastic dynamics of complex systems (World Scientific, New York, 2013).

[10] D. J. Wilkinson, Nat. Rev. Gen. 10, 122 (2009).

[11] D. Most, D. Keles, Eur. J. Op. Res. 207, 543 (2010).

[12] P. E. Smouse, S. Focardi, et al., Phyl. Trans. Roy. Soc. B 365, 2201 (2010).

[13] O. Astafiev, Yu. A. Pashkin, et al., Phys. Rev.Lett. 93, 267007 (2004).

[14] Y. M. Galperin, B. L. Altshuler, et al., Phys. Rev. Lett. 96, 097009 (2006).

[15] B. Abel, F. Marquardt, Phys. Rev. B 78, 201302(R) (2008).

[16] L.T. Kenfack, M. Tchoffo, et al., arXiv preprint arXiv:1707.02762 (2017).

[17] F. Buscemi, P. Bordone, Phys. Rev. A. 87, 042310 (2013).

[18] L.T. Kenfack, M. Tchoffo, et al., Eur. Phys. J. Plus. 132, 91 (2017).

[19] M. Tchoffo, L.T. Kenfack, et al., Eur. Phys. J. Plus. 131, 380 (2016).

[20] T.K. Lionel, T. Martin, et al., Int. J. Mod. Phys. B 30, 1750046 (2016).

[21] L.T. Kenfack, M. Tchoffo, et al., Quantum Inf Process 17, 76 (2018).

[22] L.T. Kenfack, M. Tchoffo, et al., Physica B 511, 123 (2017).

[23] L.T. Kenfack, M. Tchoffo, et al., Int. J. Quant. Inf. 15, 1750038 (2017).

[24] C. Benedetti, F. Buscemi, P. Bordone, M.A.G. Paris, Phys. Rev. A. 87, 052328 (2013).

[25] C. Benedetti, M.G.A. Paris, et al., Time-evolution of entanglement and quantum discord of bipartite systems subject to $1 / f^{\alpha}$ noise, in Proceedings of the 22nd International Conference on Noise and Fluctuations (ICNF), Montpellier, DOI:10.1109/ICNF, 6578952 (2013).

[26] C. Benedetti, F. Buscemi, et al., . Int. J. Quant. Inf. 10, 1241005 (2012).

[27] A. Basit, H. Ali, et al., Commun. Theor. Phys. 68, 29 (2017).

[28] G. Karpat, Z. Gedik,Physics Letters A 375, 4166 (2011).

[29] F. Altintas, R. Eryigit,Physics Letters A 374, 4283 (2010).

[30] D. Zhou, A. Lang, R. Joynt, Quantum Inf. Process. 9, 727 (2010).

[31] B. Corn, T. Yu, Quantum Inf Process 8, 565 (2009).

[32] T. Yu, J.H. Eberly, Optics Communications 264, 393 (2006).

[33] A. De, A. Lang, et al., Phys. Rev. A 83, 042331 (2011).

[34] J. Helm, W. T. Strunz, Phys. Rev. A 80, 042108 (2009).

[35] J. Helm, W. T. Strunz, et al., Phys. Rev. A 83, 042103 (2011).

[36] W. M. Witzel, K. Young, S. Das Sarma, Phys. Rev. B 90, 115431 (2014).

[37] W.T. Strunz, L. Díosi, N. Gisin, Phys. Rev. Lett. 82, 1801 (1999).

[38] J.T. Stockburger, H. Grabert, Phys. Rev. Lett. 88, 170407 (2002).

[39] D. Crow, R. Joynt, Phys. Rev. A 89, 042123 (2014).

[40] O. Gühne, G. Tóth, Physics Reports 474, 1 (2009).

[41] C. Sabin, G. Garcia-Alcaine, Eur. Phys. J. D. 48, 435 (2008). 